\title{
Improvement of minimally invasive parathyroidectomy outcomes by real time ultrasonography performed by a surgeon and radiologist team.
}

\author{
Omer Uslukaya1, Metehan Gumus ${ }^{1}$, Bekir Tasdemir², Cemil Goya ${ }^{3}$, Faruk Kilinc ${ }^{4}$, \\ Abdullah Oguz ${ }^{1}$, Ahmet Turkoglu ${ }^{1}$, Zubeyir Bozdag1
}

${ }^{1}$ Departmant of General Surgery, ${ }^{2}$ Department of Nuclear Medicine, ${ }^{3}$ Departmant of Radiology Dicle, ${ }^{4}$ Department of Endocrinology, Dicle University Medical Faculty, Diyarbakir, Turkey

\begin{abstract}
Aims: Minimally invasive parathyroidectomy (MIP) has become the first line of treatment for primary hyperparathyroidism caused by solitary parathyroid adenoma. In order to increase the sensitivity of high-resolution ultrasonography (hUS), surgeon performed ultrasonography (SUS) has been increasingly used preoperatively. However, a radiologist and surgeon performing ultrasonography (RSUS) has not been a usual practice. In this study, we aimed to evaluate the clinical contribution of RSUS on MIP. Material and methods: From 2012 to 2014, a total of 30 consecutive patients ( 4 male, 26 female, mean age $48.87 \pm 14.52$ years) with solitary parathyroid adenoma, were included in the study. All patients underwent preoperative hUS and Technetium-99m sestamibi scintigraphy. In patients, demographic characteristics, diagnostic tools used, levels of biochemical parameters, duration of operation, and length of hospital stay were recorded. Results: Adenomas were successfully localized by US in all patients and the surgical approach was determined according to this localization. Parathyroidectomy with MIP was successfully performed under local anesthesia in all patients. Mean operation time was $19.87 \pm 3.35$ min. Postoperative PTH and calcium values were significantly decreased. All patients were discharged from the hospital in the same day. None of the patients had complications such as recurrent laryngeal nerve injury, hematoma, or injury to nearby organs. None of the patients had drains placed. Conclusions: Adenoma is well localized by US and thus, MIP can be completed under local anesthesia. US provides a very important clinical contribution to the success of MIP. In addition to these, RSUS helps in determining the location of the incision and the shortest way to achieve the lesion; therefore, it provides a small incision and shortens duration of the operation with a minimal dissection.
\end{abstract}

Keywords: parathyroid adenoma, ultrasound, preoperative procedure, minimal access parathyroidectomy

\section{Introduction}

The incidence of primary hyperparathyroidism (pHPT) is increasing and is currently estimated to occur in 1:500 women and 1:2,000 men [1]. The traditional surgical approach is the bilateral neck exploration of all four parathyroid glands [2-4] with success rates exceeding $95 \%$ and minimal morbidity $[2,5]$. However, pHPT is caused by a solitary adenoma in $85-90 \%$ of patients

Received 03.04.2015 Accepted 27.05.2015

Med Ultrason

2015, Vol. 17, No 3, 315-321

Corresponding author: Assist. Prof. Omer Uslukaya

Department of General Surgery, Dicle University School of Medicine, Diyarbakir 21280, Turkey

Phone: +90 5052247991

Fax: +90 4122488001

Email: druslukaya@gmail.com
$[2,6,7]$. With developing technologies such as parathyroid Technetium-99 m sestamibi scintigraphy (SS) and high-resolution ultrasonography (hUS), minimally invasive parathyroidectomy (MIP) seems to be the first choice.

MIP can be defined as the ability of the surgeon to perform traditional surgical procedures in novel ways to minimize the trauma of the surgical exposure [8,9]. Given the safety and high success rate of bilateral neck exploration, the potential advantages of MIP are: decreased operating times (OT), decreased hospitalization time, reduced level of physical invasiveness, and better cosmetic results [8]. However, the success of MIP depends on accurate preoperative localization of parathyroid adenomas [10-12].

hUS and SS are currently the most widely used preoperative localization modalities. The sensitivity of hUS 
and SS identification of parathyroid adenomas ranges between $70 \%$ and $90 \%[4,13,14]$. Although the sensitivity of hUS and SS are high for solitary parathyroid adenomas, negative results of imaging studies are inevitable $[4,15,16]$. hUS is favored because of its low cost, convenience, lack of radiation exposure, and ability to evaluate concomitant thyroid nodules; however, it may fail to detect ectopic parathyroid adenomas $[4,14,17]$. Also, it has the disadvantage of being operator-dependent, with sensitivities ranging from $20 \%$ to $79 \%$ among sonographers, depending on skill, experience, and interest [18-20]. In order to increase the sensitivity of hUS, surgeon-performed ultrasonography (SUS) has been increasingly used preoperatively, and has been shown to be highly accurate in localizing abnormal glands [21-24]. However, to the best of our knowledge, the effectiveness of radiologist and surgeon performed ultrasonography (RSUS) before MIP has not been evaluated in any studies yet.

In this study, we aim to evaluate the effect of RSUS on the success rate of finding adenoma, operating time, conversion to the general anesthesia, and complications.

\section{Material and methods}

From January 2012 to October 2014, a total of 98 consecutive patients were operated at Dicle University for $\mathrm{pHPT}$. In 30 patients the pHPT was caused by solitary parathyroid adenoma and these patients were included in the study. All the patients had biochemically confirmed pHPT and single gland disease supported by imaging methods such as SS and hUS. Positive results seen on imaging modalities was used for the selection of glands to be excised. Parathyroidectomy with local anesthesia and intravenous sedation for well-localized solitary gland was preferred for patients who had no contraindication for intravenous sedation. Exclusion criteria were suspicion of multiple gland disease, multiple endocrine neoplasm syndrome or parathyroid carcinoma, non-cooperative patients who were requesting general anesthesia and preoperatively diagnosed intratyroidal parathyroid (fig 1a) or thyroid disease requiring thyroidectomy. No intraoperative parathyroid hormone assay was used after excision of the gland. All excised glands weight and sizes were measured after removal. All removed specimens were fixed in a $10 \%$ formalin solution and sent to pathology. In this study frozen section was not used and operation was finalized after excision of the gland. Biochemical values of all patients were recorded. Parathyroid hormone (PTH), total serum calcium phosphor levels of all patients were measured preoperatively. These levels were measured again postoperatively in the first day, 2nd week, 3rd month, and 6th month.

The demographic characteristics of the patients, operation time (OT), and hospitalization time were retrospectively recorded from the hospital database.

\section{Ultrasound technique}

Combined RSUS (RUS + SUS) took place in the presence of both (surgeon + radiologist) in the Radiology Department, one day before surgery. Patients were scanned in supine position with neck in hyperextension. A complete cervical ultrasound with imaging of the thyroid, parathyroid glands, vascular structures, and any associated lymphadenopathy was documented. The incision site wasdetermined and marked under US guidance. The anatomic location and dimensions of suspected abnormal parathyroid glands were recorded.

\section{Anesthetic technique}

Intravenous $0.02 \mathrm{mg} / \mathrm{kg}$ midazolam was given in the preoperative room then patients were moved into the operation theatre and $1 \mu \mathrm{g} / \mathrm{kg}$ fentanyl IV was given after monitorization. A mixture of $1 \mathrm{mg} / \mathrm{kg} 0.25 \%$ bupivacaine and $1 \mathrm{mg} / \mathrm{kg} 1 \%$ lidocaine was prepared and half of the dose was used on the incision area. The other half was used for the infiltration of the deeper levels and neighboring tissues. If patients complained of pain, the sedation of the patient was increased with $0.5 \mathrm{mg} / \mathrm{kg}$ propofol. If by any reason conversion to general anesthesia was required the patients were intubated by the anesthesiologists.

\section{Surgical technique}

MIP was performed through a 3-3.5 cm curvilinear transverse collar incision approximately $2-3 \mathrm{~cm}$ above the sternal notch. We used two different approaches (anterior or lateral approach) in operations, whichever is appropriate (fig 1b). The type of approach was decided before with RSUS (fig 1c, d). In the anterior approach or midline approach, the plane between the strap muscles was used. In the lateral approach, or back-door approach, the plane between the strap muscles medially and the carotid sheath laterally was used. Operation time was defined as the duration from the incision made and up to stitching of the skin.

\section{Statiscal analyses}

Statistical analyses were performed using SPSS software program version 15.0 for Windows (SPSS Inc., Chicago, IL, USA). Categorical variables were expressed as counts and percentages, while continuous variables were expressed as mean \pm standard deviation or range. Differences between baseline and postoperative $\mathrm{Ca}, \mathrm{P}$ and PTH values were tested using paired samples t-test or Wilcoxon rank test after determining normality according to Kolmogorov-Smirnov test. A p value $<0.05$ was considered statistically significant. 


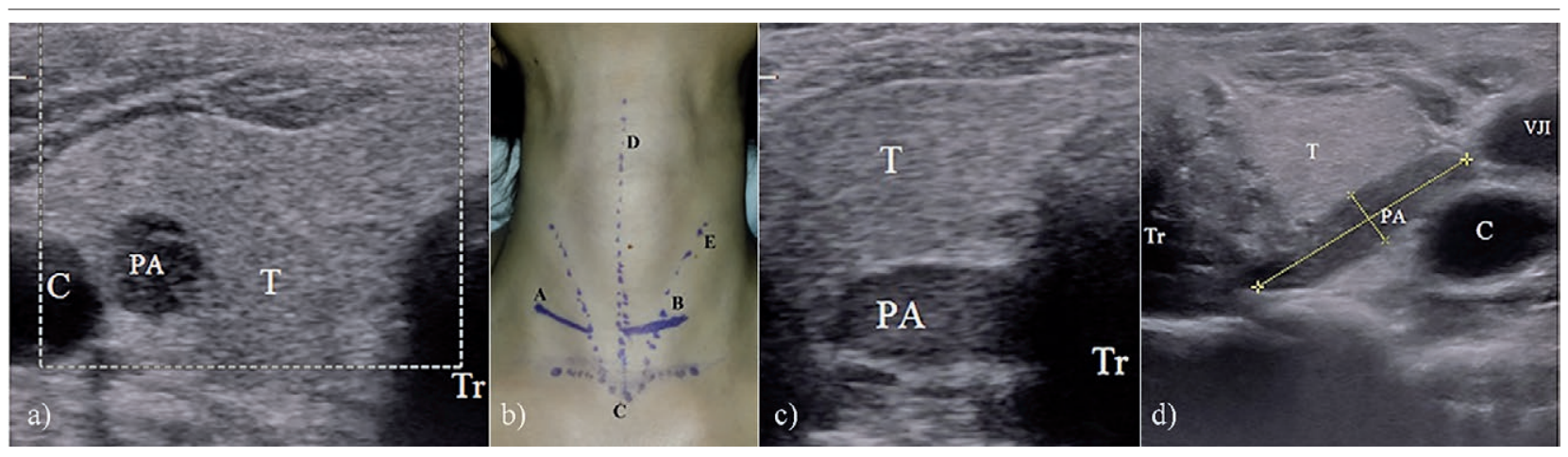

Fig 1. a) Round hypoechoic parathyroid adenoma completely surrounded by the right lobe of the thyroid, which is not suitable for minimally invasive parathyroidectomy; b) Approaches to Parathyroid Gland. Two incision types used for minimally invasive parathyroidectomy (A - Incision for lateral approach, B - Incision for medial approach, C - Sternal notch, D - Midline, E - Anterior border of sternocleidomastoid muscle); c) Parathyroid adenoma adjacent to lateral of trachea and posterior aspect of the inferior portion of right lobe of thyroid gland, which is proper for anterior approach; d) Parathyroid adenoma anterior to common carotid artery and internal jugular vein, posterior to the left lobe of thyroid gland, which is proper for lateral approach. C - Arteria carotis communis, PA - Parathyroid adenoma, T - Thyroid gland, Tr - Trachea, VJI -Vena jugularis interna

\section{Results}

Of the 30 patients, $26(86.7 \%)$ were female. The average age of the patients was $48.87 \pm 14.52$ years (range 19-75). The distribution of excised adenomas was $63.3 \%$ $(\mathrm{n}=19)$ in the right side and $36.7 \%(\mathrm{n}=11)$ in the left side. Intervention with local anesthesia was applied to all patients. There was no patients with body mass index (BMI) higher than 30 . None of the patients required conversion to general anesthesia.

All patients had biochemically confirmed pHPT and single gland disease supported by imaging methods, SS and/or hUS. hUS and SS were performed in all patients. While parathyroid adenomas were detected with hUS in all patients, SS was unable to identify parathyroid adeno$\mathrm{ma}$ in two $(6.7 \%)$ patients. According to hUS and operative findings, localization of parathyroid adenomas was $18(60.0 \%)$ in right lower zone, $1(3.3 \%)$ in right upper zone, $9(30.0 \%)$ in left lower zone, and $2(6.7 \%)$ in left upper zone. All adenomas detected with SS were in concordance with hUS and operation localization.

The average weight in excised parathyroid adenomas was $3.44 \pm 4.43 \mathrm{gr}$ (range $0.5-17.6 \mathrm{gr}$ ), while the average of the largest diameter was $2.08 \pm 0.99 \mathrm{~cm}$ (range $0.8-4.5$ $\mathrm{cm})$. Preoperative and postoperative mean serum total calcium, phosphorus, and PTH values are detailed in table I. While postoperative $\mathrm{Ca}$ and PTH levels were significantly lower than preoperative values $(p<0.001)$, the phosphorus value was significantly higher $(\mathrm{p}=0.015)$.

All patients were followed-up for at least 6 months. According to biochemical results (serum PTH, total calcium and serum phosphorus), cure was achieved in all patients. This indicates a successful operation.

Mean operation time was $19.87 \pm 3.35$ min (range 15$28 \mathrm{~min})$.

All patients were discharged from the hospital or transferred to the Endocrinology Department in the same day.

None of the patients had complications such as recurrent laryngeal nerve injury, hematoma, or injury to nearby organs.

\section{Discussions}

The technical success rate of MIP was $98 \%$ in the study of Udelsman [25]. This rate reached $100 \%$ in our study. The normalization of calcium levels in parathyroid surgery shows the success of the operation. But it should not be considered as the single indicator of the success. Surgery should also be performed with minimal morbid-

Table I. Biochemical results of patients.

\begin{tabular}{lllll}
\hline Parameters & Preoperative & $\begin{array}{l}\text { Postoperative } \\
\text { 1st day }\end{array}$ & $\begin{array}{l}\text { Postoperative } \\
\text { 6th month }\end{array}$ & Normal range \\
\hline PTH & $749.22 \pm 998.04$ & $24.31 \pm 15.47$ & $56.73 \pm 17.26$ & $15-65 \mathrm{pg} / \mathrm{ml}$ \\
Calcium & $11.91 \pm 1.89$ & $8.51 \pm 0.91$ & $9.13 \pm 0.89$ & $8.4-10.2 \mathrm{mg} / \mathrm{dl}$ \\
Phosphor & $2.50 \pm 0.67$ & $2.88 \pm 0.67$ & $3.60 \pm 0.61$ & $3.2-5.5 \mathrm{mg} / \mathrm{dl}$ \\
\hline
\end{tabular}

PTH: Parathyroid hormone 
ity, no mortality, low recurrence rates, and a reasonable cost [26]. MIP prevents unnecessary mobilization of neck structures, thereby lowering the rate of recurrent laryngeal nerve damage $[25,27]$. The solitary adenoma causes $85-90 \%$ of all pHPT [27-29]. For single gland disease, MIP can be applied securely and efficiently with local anesthesia and intravenous (IV) sedation [30-33]. In a large number of centers, MIP can now be routinely performed as an outpatient procedure [25,34-37]. However, it can be sufficient if accurate preoperative localization is performed $[2,3,6,38,39]$. The combination of SS and neck hUS has a $74-90 \%$ sensitivity in localizing a parathyroid adenoma $[18,19,40,41]$.

Nowadays, hUS is increasingly being used. Performing a hUS before the patient undergoes MIP is beneficial as it enables exact localization of the pathologic parathyroid. It is highly sensitive in detecting parathyroid adenomas located behind the thyroid gland or beyond the lower contour of the thyroid. It also enables the identification and evaluation of any concomitant thyroid disease prior to surgery. Recently, SUS has been increasingly used as a complementary imaging study to SS [42-44]. It has shown that SUS is as good as RUS in localizing single gland disease in patients with sporadic pHTP. The sensitivity of SUS in localizing parathyroid adenomas has been reported to be $67 \%-87 \%$ [18,22,45-47]. Steward et al [46] reported one of the highest success rates for SUS: a sensitivity of $91 \%$. The rate of accuracy results of previous studies are detailed in Table II.

When US is performed by the operating surgeon it allows the surgeon to directly visualize the parathyroid adenoma and take real-time images, in multiple planes, in the preoperative setting and correlate it with the thyroid and other landmarks in the neck. [42]. Also, it permits the planning of the neck incision, thereby increasing the safety of the operation [52]. Therefore, we think that the time spent for surgery following RSUS is shorter than that for surgery following RUS, showing that combination of the surgery with RSUS is effective. In patients with morbid obesity, surgical procedure is much more challenging in terms of operation time, length of incision, seroma, drainage. In our study, all patients had BMI under 30.

Radiologist's role cannot be denied in detecting parathyroid adenoma. For example, in one case the adenoma within carotid sheath was not determined by SUS, but was easily found with RUS. In this study, we showed that when an experienced radiologist, along with a surgeon, perform USpreoperatively, solitary adenomas in all the patients were found easily and accurately. It is important to have an available radiologist on site and demand. There is a learning curve associated with SUS. Arora et al [48] mentioned that they confirmed their SUS with MIBI for the first 250 patients, to complete the learning curve. In our method we think that only ten cases are enough for a learning curve, because we perform US in the presence of a radiologist, not a surgeon alone. While MIP was completed successfully in $84 \%$ of patients (under local anesthesia) in one study [31], the completion rate was $100 \%$ for us. One should consider that proper patient selection and the experience of the surgical team play an important role in the conversion rate. This characteristic explains in part the very low conversion rate reported in larger series $(0.9-8 \%)[53,54]$. In our study we have no case requiring conversion to general anesthesia, due to the proper selection of the cases.

While Kwon et al [2], determined the localization of parathyroid tumorby examining preoperative documents,

Table II. Comparison of success of localization methods in primary hyperparathyroidism.

\begin{tabular}{lllllll}
\hline \multirow{2}{*}{ Author } & Year & $\mathbf{n}$ & Method (the success rate) & \\
& & & RUS n(\%) & SUS n(\%) & SS n(\%) & US+SS n(\%) \\
\hline Soon et al [18] & 2008 & 218 & $148(82.76)$ & $87(81.76)$ & & \\
Deutmeyer et al [21] & 2011 & 220 & & $96(88)$ & & \\
Aliyev et al [24] & 2014 & 1000 & & $842(84)$ & $842(82.5)$ & \\
Gilat et al [27] & 2004 & 77 & $77(85.72)$ & & & \\
Teksoz et al [30] & 2013 & 42 & $15(35.71)$ & & $11(26.9)$ & $16(38.09)$ \\
Untch et al [42] & 2011 & 516 & $503(76)$ & $392(87)$ & & \\
Steward et al [46] & 2006 & 106 & $106(42)$ & $106(82)$ & $106(44)$ & \\
Jabiev et al [47] & 2009 & 442 & & $52(88.5)$ & & $390(74.9)$ \\
Arora et al [48] & 2009 & 160 & & $160(74)$ & $119(56)$ & \\
Livingston et al [49] & 2008 & 100 & & $18(94)$ & $79(79)$ & \\
Hughes et al [50] & 2014 & 1910 & & $1644(80)$ & $1165(88)$ & $920(88)$ \\
Prasannan et al [51] & 2007 & 130 & & $103(79.2)$ & $102(78.4)$ & \\
\hline
\end{tabular}

RUS: radiologist performed ultrasonography, SUS:surgeon-performed ultrasound, SS: technetium-99m sestamibi scintigraphy, US: ultrasonography. $n$ : the number of patients 
we evaluate this in real-time. While radiologists perform hUS in the supine position, radiologists and surgeons perform hUS in supine position with hyperextension which is the position used in surgery. This position provides the surgeon the location of the adenoma and its relation to neighboring structures such as surrounding vasculature and the trachea. It also provides the nearest incision localization and distance from skin to adenoma. This leads to less tissue damage and a shorter operation time. Anatomical localization facilitates skin incision placement by the lateral mini-incision technique and improves cosmetic results [2,5]. MIP are carried out through a $2-4 \mathrm{~cm}$ incision [8,34].

Deutmeyer et al [21] performed operations under general anesthesia by intraoperative PTH monitoring based on SUS localization and showed that SS adds little additional information to guide surgery. Some authors do not advocate MIP with hUS as the only preoperative investigation study because the size of a parathyroid gland does not correlate with the function [18,55]. Using hUS localization alone, it is therefore possible to remove an enlarged nonfunctioning parathyroid gland and miss the smaller pathological gland [18]. However, in our study, SS could not detect adenoma in two patients and it did not provide additional information to hUS in our other patients. Therefore, we suggest using SS only for the cases with unclear hUS findings. In this situation the radiation exposure is avoided and can save money and time.

Black et al [56] suggested that although local/cervical block anesthesia and general anesthesia groups were equivalent for experienced complications, the advantage of local/cervical block anesthesia vs general anesthesia relates to the decreased operating time, costs, and need for oral pain medication. Compared to general anesthesia, local anesthesia [57] and hypnosedation [58] decrease rates of nausea and vomiting, resulting in decreased postoperative bleeding. Additionally, endotracheal intubation by itself has been associated with objective functional voice changes in as much as $5 \%$ of patients [59]. On the contrary, several studies show that general anesthesia give more comfort for surgeon while dissecting the tissues, leading to a reduced risk of hemorrhage $[59,60]$. Local anesthesia and hypnosedation is especially appropriate for ASA III or IV anesthetic risk category [8]. Furthermore, avoidance of general anesthesia and/or a hospital admission for the vast majority of patients results in significant cost savings.

A focused anterior approach, which is the most commonly used technique for MIP, includes lateral retraction or sometimes splitting of the strap muscles. Many studies about MIP mention the anterior approach [30-32]. In the lateral approach the gland is reached by splicing the anterior wall of the sternocleidomastoid muscle and lateral border of the strap muscles via a small skin incision $[30,33]$. In this study, we think that decision of surgical approaches (anterior or lateral) by RSUS provided an important benefit in duration and feasibility of the surgery.

MIP shortens the operative and anesthesia time $[27,29,61]$. In literature the mean operation time of MIP ranges from 15 to 56 minutes [30,31]. It also shortens the hospitalization time [25]. In this study, duration of the surgery was short $(\sim 20 \mathrm{~min})$ and patients were not hospitalized longer than one day.

There are several limitations to this study. First, the ultrasonographers, both radiologists and surgeons, were not blinded to the results of SS. Second, this was a retrospective study that did not have a control group. A larger study (necessarily randomized, multicentric) would be better in order to assess the results after RUS (branch 1), SUS (branch 2) and RSUS (branch 3). Finally, the small number of patients that were enrolled in the study was a limitation.

\section{Conclusions}

The success of MIP depends on the success in localization and identification of abnormal glands. In MIP, the incision is small, dissection is minimal, postoperative pain is less, and hospital stay is shorter. These procedures may be done as an outpatient surgery, even with local anesthesia. As a result, it is important that surgeons join hUS, due to the selection of patient and successful treatment of selected patients. We think that surgeons and radiologists are complementary to each other. Moreover, our success which was $100 \%$, stems from RSUS. If patients require a second operation, the anatomical structures of the region will be intact. Use of local anesthesia avoids complications associated with general anesthesia.

\section{References}

1. Grosso I, Sargiotto A, D'Amelio P, et al. Preoperative localization of parathyroid adenoma with sonography and $99 \mathrm{mTc}$-sestamibi scintigraphy in primary hyperparathyroidism. J Clin Ultrasound 2007; 35: 186-190.

2. Kwon JH, Kim EK, Lee HS, Moon HJ, Kwak JY. Neck ultrasonography as preoperative localization of primary hyperparathyroidism with an additional role of detecting thyroid malignancy. Eur J Radiol 2013; 82: e17-e21.

3. Patel CN, Salahudeen HM, Lansdown M, Scarsbrook AF. Clinical utility of ultrasound and 99mTc sestamibi SPECT/ CT for preoperative localization of parathyroid adenoma in patients with primary hyperparathyroidism. Clin Radiol 2010; 65: 278-287. 
4. Erbil Y, Salmaslioglu A, Kabul E, et al. Use of preoperative parathyroid fine-needle aspiration and parathormone assay in the primary hyperparathyroidism with concomitant thyroid nodules. Am J Surg 2007; 193: 665-671.

5. Barczynski M, Golkowski F, Konturek A, et al. Technetium-99m-sestamibi subtraction scintigraphy vs. ultrasonography combined with a rapid parathyroid hormone assay in parathyroid aspirates in preoperative localization of parathyroid adenomas and in directing surgical approach. Clin Endocrinol (Oxf) 2006; 65: 106-113.

6. Erbil Y, Barbaros U, Salmaslioglu A, et al. Value of parathyroid hormone assay for preoperative sonographically guided parathyroid aspirates for minimally invasive parathyroidectomy. J Clin Ultrasound 2006; 34: 425-429.

7. Maser C, Donovan P, Santos F, et al. Sonographically guided fine needle aspiration with rapid parathyroid hormone assay. Ann Surg Oncol 2006; 13: 1690-1695.

8. Henry JF. Minimally invasive thyroid and parathyroid surgery is not a question of length of the incision. Langenbecks Arch Surg 2008; 393: 621-626.

9. Roth SI, Wang CA, Potts JT Jr. The team approach to primary hyperparathyroidism. Hum Pathol 1975; 6: 645-648.

10. Erbil Y, Barbaros U, Tükenmez M, et al. Impact of adenoma weight and ectopic location of parathyroid adenoma on localization study results. World J Surg 2008; 32: 566-571.

11. Sackett WR, Barraclough B, Reeve TS, Delbridge LW. Worldwide trends in the surgical treatment of primary hyperparathyroidism in the era of minimally invasive parathyroidectomy. Arch Surg 2002; 137: 1055-1059.

12. Siperstein A, Berber E, Mackey R, Alghoul M, Wagner K, Milas M. Prospective evaluation of sestamibi scan, ultrasonography, and rapid PTH to predict the success of limited exploration for sporadic primary hyperparathyroidism. Surgery $2004 ; 136$ : 872-880.

13. Smith JR, Oates ME. Radionuclide imaging of the parathyroid glands: patterns, pearls, and pitfalls. Radiographics 2004; 24: 1101-1115.

14. Huppert BJ, Reading CC. Parathyroid sonography: imaging and intervention. J Clin Ultrasound 2007; 35: 144-155.

15. Lo CY, Lang BH, Chan WF, Kung AW, Lam KS. A prospective evaluation of preoperative localization by technetium99m sestamibi scintigraphy and ultrasonography in primary hyperparathyroidism. Am J Surg 2007; 193: 155-159.

16. Mihai R, Gleeson F, Buley ID, Roskell DE, Sadler GP. Negative imaging studies for primary hyperparathyroidism are unavoidable: correlation of sestamibi and high-resolution ultrasound scanning with histological analysis in $150 \mathrm{pa}-$ tients. World J Surg 2006; 30: 697-704.

17. Barraclough BM, Barraclough BH. Ultrasound of the thyroid and parathyroid glands. World J Surg 2000; 24: 158-165.

18. Soon PS, Delbridge LW, Sywak MS, Barraclough BM, Edhouse P, Sidhu SB. Surgeon performed ultrasound facilitates minimally invasive parathyroidectomy by the focused lateral mini-incision approach. World J Surg 2008; 32: 766-771.

19. Purcell GP, Dirbas FM, Jeffrey RB, et al. Parathyroid localization with high-resolution ultrasound and technetium Tc 99 m sestamibi. Arch Surg 1999; 134: 824-828.
20. Lloyd MN, Lees WR, Milroy EJ. Pre-operative localisation in primary hyperparathyroidism. Clin Radiol 1990; 41: 239-243.

21. Deutmeyer C, Weingarten M, Doyle M, Carneiro-Pla D. Case series of targeted parathyroidectomy with surgeonperformed ultrasonography as the only preoperative imaging study. Surgery 2011; 150: 1153-1160.

22. Solorzano CC, Carneiro-Pla DM, Irvin GL 3rd. Surgeonperformed ultrasonography as the initial and only localizing study in sporadic primary hyperparathyroidism. J Am Coll Surg 2006; 202: 18-24.

23. Haber RS, Kim CK, Inabnet WB. Ultrasonography for preoperative localization of enlarged parathyroid glands in primary hyperparathyroidism: comparison with (99m) technetium sestamibi scintigraphy. Clin Endocrinol (Oxf) 2002; 57: 241-249.

24. Aliyev S, Agcaoglu O, Aksoy E, et al. An analysis of whether surgeon-performed neck ultrasound can be used as the main localizing study in primary hyperparathyroidism. Surgery 2014; 156: 1127-1131.

25. Udelsman R. Six hundred fifty-six consecutive explorations for primary hyperparathyroidism. Ann Surg 2002; 235: 665-670.

26. Mariani G, Gulec SA, Rubello D, et al. Preoperative localization and radioguided parathyroid surgery. J Nucl Med 2003; 44: 1443-1458.

27. Gilat H, Cohen M, Feinmesser R, et al. Minimally invasive procedure for resection of a parathyroid adenoma: the role of preoperative high-resolution ultrasonography. J Clin Ultrasound 2005; 33: 283-287.

28. Song AU, Phillips TE, Edmond CV, Moore DW, Clark SK. Success of preoperative imaging and unilateral neck exploration for primary hyperparathyroidism. Otolaryngol Head Neck Surg 1999; 121: 393-397.

29. Smit PC, Borel Rinkes IH, van Dalen A, van Vroonhoven TJ. Direct, minimally invasive adenomectomy for primary hyperparathyroidism. An alternative to conventional neck exploration? Ann Surg 2000; 231: 559-565.

30. Teksoz S, Bukey Y, Ozcan M, Arikan AE, Erbabacan SE, Ozyegin A. Minimal invasive parathyroidectomy with local anesthesia for well-localized primary hyperparathyroidism: “Cerrahpasa experience”. Updates Surg 2013; 65: 217-223.

31. Cohen MS, Finkelstein SE, Brunt LM, et al. Outpatient minimally invasive parathyroidectomy using local/regional anesthesia: a safe and effective operative approach for selected patients. Surgery 2005; 138: 681-687.

32. Shindo ML, Rosenthal JM, Lee T. Minimally invasive parathyroidectomy using local anesthesia with intravenous sedation and targeted approaches. Otolaryngol Head Neck Surg 2008; 138: 381-387.

33. Shindo ML, Rosenthal JM. Minimal access parathyroidectomy using the focused lateral approach: technique, indication, and results. Arch Otolaryngol Head Neck Surg 2007; 133: $1227-1234$.

34. Udelsman R, Donovan PI. Remedial parathyroid surgery: changing trends in 130 consecutive cases. Ann Surg 2006; 244: 471-479. 
35. Irvin GL 3rd, Sfakianakis G, Yeung L, et al. Ambulatory parathyroidectomy for primary hyperparathyroidism. Arch Surg 1996; 131: 1074-1078.

36. Lo Gerfo P. Bilateral neck exploration for parathyroidectomy under local anesthesia: a viable technique for patients with coexisting thyroid disease with or without sestamibi scanning. Surgery 1999; 126: 1011-1015.

37. Chen H, Mack E, Starling JR. A comprehensive evaluation of perioperative adjuncts during minimally invasive parathyroidectomy: which is most reliable? Ann Surg 2005; 242: 375-380.

38. Levy JM, Kandil E, Yau LC, Cuda JD, Sheth SN, Tufano RP. Can ultrasound be used as the primary screening modality for the localization of parathyroid disease prior to surgery for primary hyperparathyroidism? A review of 440 cases. ORL J Otorhinolaryngol Relat Spec 2011; 73: 116-120.

39. Ruda JM, Hollenbeak CS, Stack BC Jr. A systematic review of the diagnosis and treatment of primary hyperparathyroidism from 1995 to 2003 . Otolaryngol Head Neck Surg 2005; 132: 359-372.

40. Lumachi F, Ermani M, Basso S, Zucchetta P, Borsato N, Favia $\mathrm{G}$. Localization of parathyroid tumours in the minimally invasive era: which technique should be chosen? Population-based analysis of 253 patients undergoing parathyroidectomy and factors affecting parathyroid gland detection. Endocr Relat Cancer 2001; 8: 63-69.

41. Geatti O, Shapiro B, Orsolon PG, et al. Localization of parathyroid enlargement: experience with technetium-99 m methoxyisobutylisonitrile and thallium-201 scintigraphy, ultrasonography and computed tomography. Eur J Nucl Med 1994; 21: 17-22.

42. Untch BR, Adam MA, Scheri RP, et al. Surgeon-performed ultrasound is superior to 99Tc-sestamibi scanning to localize parathyroid adenomas in patients with primary hyperparathyroidism: results in 516 patients over 10 years. J Am Coll Surg 2011; 212: 522-529.

43. Miller BS, Gauger PG, Broome JT, Burney RE, Doherty GM. An international perspective on ultrasound training and use for thyroid and parathyroid disease. World J Surg 2010; 34: 1157-1163.

44. Davis ML, Quayle FJ, Middleton WD, et al. Ultrasound facilitates minimally invasive parathyroidectomy in patients lacking definitive localization from preoperative sestamibi scan. Am J Surg 2007; 194: 785-790.

45. Van Husen R, Kim LT. Accuracy of surgeon-performed ultrasound in parathyroid localization. World J Surg 2004; 28: 1122-1126.

46. Steward DL, Danielson GP, Afman CE, Welge JA. Parathyroid adenoma localization: surgeon-performed ultrasound versus sestamibi. Laryngoscope 2006; 116: 1380-1384.

47. Jabiev AA, Lew JI, Solorzano CC. Surgeon-performed ultrasound: a single institution experience in parathyroid localization. Surgery 2009; 146: 569-575.

48. Arora S, Balash PR, Yoo J, Smith GS, Prinz RA. Benefits of surgeon-performed ultrasound for primary hy- perparathyroidism. Langenbecks Arch Surg 2009; 394: 861-867.

49. Livingston CD, Victor B, Askew R, et al. Surgeon-performed ultrasonography as an adjunct to minimally invasive radio-guided parathyroidectomy in 100 consecutive patients with primary hyperparathyroidism. Endocr Pract 2008; 14: 28-32.

50. Hughes DT, Sorensen MJ, Miller BS, Cohen MS, Gauger PG. The biochemical severity of primary hyperparathyroidism correlates with the localization accuracy of sestamibi and surgeon-performed ultrasound. J Am Coll Surg 2014; 219: 1010-1019.

51. Prasannan S, Davies G, Bochner M, Kollias J, Malycha P. Minimally invasive parathyroidectomy using surgeonperformed ultrasound and sestamibi. ANZ J Surg 2007; 77: 774-777.

52. Soon PS, Yeh MW, Sywak MS, Roach P, Delbridge LW, Sidhu SB. Minimally invasive parathyroidectomy using the lateral focused miniincision approach: is there a learning curve for surgeons experienced in the open procedure? J Am Coll Surg 2007; 204: 91-95.

53. Bellantone R, Raffaelli M, DE Crea C, Traini E, Lombardi CP. Minimally-invasive parathyroid surgery. Acta Otorhinolaryngol Ital 2011; 31: 207-215.

54. Miccoli P, Berti P, Materazzi G, Massi M, Picone A, Minuto MN. Results of video-assisted parathyroidectomy: single Institution's six year experience. World J Surg 2004; 28 : 1216-1218.

55. Mun HC, Conigrave A, Wilkinson M, Delbridge L. Surgery for hyperparathyroidism: does morphology or function matter most? Surgery 2005; 138: 1111-1120.

56. Black MJ, Ruscher AE, Lederman J, Chen H. Local/cervical block anesthesia versus general anesthesia for minimally invasive parathyroidectomy: what are the advantages? Ann Surg Oncol 2007; 14: 744-749.

57. Lo Gerfo P, Kim LJ. Technique for regional anesthesia: thyroidectomy and parathyroidectomy. In: Van Heerden JA, Farley DR, editors. Operative technique in general surgery. Surgical exploration for hyperparathyroidism. Philadelphia: WB Saunders; 1999: 95-102.

58. Meurisse M, Hamoir E, Defechereux T. Bilateral neck exploration under hypnosedation. A new standard of care in primary hyperparathyroidism. Ann Surg 1999; 229: 401-408.

59. Carling T, Donovan P, Rinder C, Udelsman R. Minimally invasive parathyroidectomy using cervical block: reasons for conversion to general anesthesia. Arch Surg 2006; 41: 401-404.

60. Monchik JM, Barellini L, Langer P, Kahya A. Minimally invasive parathyroid surgery in 103 patients with local/regional anesthesia, without exclusion criteria. Surgery 2002; 131: 502-508.

61. Ulanovski D, Feinmesser R, Cohen M, Sulkes J, Dudkiewicz M, Shpitzer T. Preoperative evaluation of patients with parathyroid adenoma: role of high resolution ultrasonography. Head Neck 2002; 24: 1-5. 